\title{
Immunohistochemical mapping of neurotensin in the alpaca diencephalon
}

\author{
Manuel Lisardo Sánchez ${ }^{1}$, Arturo Mangas ${ }^{1}$, Luis Enrique Medina1, Luis Angel Aguilar², \\ Zaida Díaz-Cabiale ${ }^{3}$, José Angel Narváez ${ }^{3}$, Rafael Coveñas ${ }^{1}$
}

\author{
${ }^{1}$ Institute of Neurosciences of Castilla y León (INCYL), Laboratory of Neuroanatomy \\ of the Peptidergic Systems (Lab. 14), Salamanca, Spain \\ ${ }^{2}$ San Ignacio de Loyola University (USIL), Faculty of Health Sciences, Lima, Peru \\ ${ }^{3}$ University of Málaga, School of Medicine, Department of Physiology, Málaga, Spain
}

\begin{abstract}
Introduction. The distribution of the immunoreactive cell bodies and fibers containing neurotensin in the alpaca diencephalon was determined by an immunohistochemical technique.

Material and methods. The study was carried out in four male alpacas that lived at sea level. Brains of deeply anesthetized animals were fixed by perfusion with $4 \%$ paraformaldehyde. Cryostat sections were stained by a standard immunohistochemical method.

Results. Cell bodies containing neurotensin were observed in the zona incerta and hypothalamus. A low/moderate density of these cell bodies was observed in the lateral hypothalamic area, anterior and dorsal hypothalamic areas, suprachiasmatic nucleus, periventricular region of the hypothalamus and in the ventromedial hypothalamic nucleus. In both thalamus and hypothalamus, immunoreactive fibers showed a widespread distribution. In the thalamus, a high density of these fibers was mainly found in the midline nuclei, whereas in the hypothalamus a high density was in general observed in the whole structure.

Conclusions. In comparison with other mammals, the thalamus of the alpaca showed the most widespread distribution of neurotensin-immunoreactive fibers. The widespread distribution of neurotensin through the alpaca diencephalon suggests that the peptide can be involved in many physiological actions. (Folia Histochemica et Cytobiologica 2018, Vol. 56, No. 1, 49-58)
\end{abstract}

Key words: camelid; Lama pacos; hypothalamus; thalamus; IHC

\section{Introduction}

Many immunohistochemical and radioimmunoassay studies have been carried out on the distribution of the tridecapeptide neurotensin in the mammalian central nervous system [1-11]. In the species studied (e.g., rat, guinea pig, cat, minipig, monkey, man), these works have reported a widespread distribution

Correspondecne address: M.L. Sánchez

University of Salamanca, Institute of Neurosciences

of Castilla y Léon (INCYL)

Laboratory of Neuroanatomy of the Peptidergic Systems (Lab.14)

c/ Pintor Fernando Gallego, 1, 37007-Salamanca, Spain

tel.: +34 923294400 ext. 1856

fax: +34923294549

e-mail: lisardosanchez8@gmail.com of neurotensin in the central nervous system and this suggests that the peptide might be involved in many physiological actions. In fact, neurotensin, acting as a neurotransmitter and/or neuromodulator, has been involved in several physiological actions affecting hemodynamic, neuroendocrine, food intake, nociceptive, thermoregulatory, locomotor, respiratory, sleep-waking, gustatory, memory, auditory and glucoregulatory systems $[1,9,12-16]$. Moreover, it is known that neurotensin promotes the dendritic spine maturation and dendrite elongation [17] and modulates high-voltage-activated calcium currents [18]. The neurotensin/neurotensin receptor system is also involved in cancer (e.g., neuroendocrine tumors, leukemia, pancreatic adenocarcinoma) [19-21]. Despite the data on the distribution of neurotensin in the mammalian central nervous system (CNS) [1-11], 
the presence of neurotensin in camelids has not been reported, with the exception of alpaca (Lama pacos), in which its presence in the brainstem was demonstrated by immunohistochemistry [12]. The alpaca is a camelid very important for some countries in South America (e.g., Peru). Due to the economic importance of the wool production, several studies have been published on the reproductive mechanisms of these animals [22-26]. Moreover, since 2007, the chemical neuroanatomy of neuropeptides in the alpaca CNS has increased considerably. Using immunohistochemical (IHC) techniques, the distribution of calcitonin gene-related peptide, somatostatin-28 (1-12), leucine-enkephalin, adrenocorticotropic hormone, beta-endorphin (1-27), alpha-melanocyte-stimulating hormone and alpha-neo-endorphin has been reported in the alpaca's CNS [27-32]. However, to date no data are currently available in the literature detailing the location of either fibers or cell bodies containing neurotensin in alpaca's diencephalon, a CNS region involved in many important functional mechanisms. Therefore, the aims of this study were to examine the distribution of immunoreactive structures containing neurotensin in the diencephalon of alpaca using immunohistochemistry.

\section{Material and methods}

Animals. Four male adult alpacas (Lama pacos) (Huacaya race) (5-8 years; 70-80 kg) were obtained from the Peruvian University Cayetano Heredia (Faculty of Veterinary Medicine and Animal Sciences, Lima, Peru). The experimental design, protocols, and procedures of this work were performed under the principles of laboratory animal care and under the guidelines of the ethical and legal recommendations of Peruvian and Spanish legislation. This work was also approved by the research commission of the Peruvian University Cayetano Heredia (Lima, Peru). The animals were kept all their life at the altitude of $0 \mathrm{~m}$ under standard conditions of light (lights on at 06:00 and off at 20.00 h) and temperature $\left(26^{\circ} \mathrm{C}\right)$, and had free access to food and water.

Tissue preparation. As previously reported [27-32], animals were deeply anesthetized with ketamine $(10 \mathrm{mg} / \mathrm{kg})$ and xylazine (4 mg/kg) (both intravenously), heparinized, and perfused via the carotid artery with 31 of cold $0.9 \% \mathrm{NaCl}$. This pre-rinse was immediately followed by infusion of the fixative: 51 of cold $4 \%$ paraformaldehyde in $0.15 \mathrm{M}$ phosphate-buffered saline (PBS) ( $\mathrm{pH}$ 7.2). The diencephalons were dissected out and post-fixed overnight in the latter solution and cryoprotected by immersion in increasing concentrations of sucrose solution (10-30\%) until they sank. Using a cryostat, 50-mm frontal sections were cut, collected in PBS and kept at $4^{\circ} \mathrm{C}$.
Immunohistochemistry. The IHC procedure has been carried out as described previously [12]. In order to avoid possible interference by endogenous peroxidase, free-floating sections were treated with a mixture of $\mathrm{NH}_{3}, \mathrm{NaOH}$ and $\mathrm{H}_{2} \mathrm{O}_{2}$ (Panreac, Barcelona, Spain) for $20 \mathrm{~min}$. Then, sections were washed in PBS $(3 \times 10 \mathrm{~min})$ and pre-incubated for $30 \mathrm{~min}$ in PBS containing $1 \%$ normal horse serum (Sigma-Aldrich, Madrid, Spain) and 0.3\% Triton X-100 (Panreac, Barcelona, Spain) in order to enhance antibody penetration. Then, sections were incubated overnight at $4^{\circ} \mathrm{C}$ in the same phosphate buffer containing the anti-neurotensin antibody (gift of Professor Gérard Tramu), diluted 1:3,000. Sections were then rinsed extensively in PBS for $30 \mathrm{~min}$ and incubated for $1 \mathrm{~h}$ at room temperature with biotinylated anti-rabbit IgG (Vector Laboratories, Burlingame, CA, USA) diluted 1:200 in the same buffer. Thereafter, sections were incubated with Vectastain ABC reagent (Vector Laboratories) for $1 \mathrm{~h}$ at room temperature. After washing the sections in PBS (30 min) and Tris-HCl buffer (Panreac, Barcelona, Spain) ( $\mathrm{pH}$ 7.6) (10 $\mathrm{min}$ ), the tissue-bound peroxidase was developed with $\mathrm{H}_{2} \mathrm{O}_{2}$, using 3, 3'-diaminobenzidine (Sigma-Aldrich) as chromogen. Finally, sections were rinsed with PBS and coverslipped with PBS/glycerol (1/1).

Specificity of the antisera. The polyclonal anti-neurotensin antibody (obtained in the laboratory of Professor Gérard Tramu, University of Bordeaux I, France) was raised in rabbits as previously reported [12]. Moreover, the immunological properties of the anti-neurotensin antiserum have been published previously $[12,33]$.

In this work, the specificity of the immunostaining was checked by: 1) preabsorption of the primary antiserum with synthetic neurotensin (Sigma-Aldrich) $(100 \mu \mathrm{g} / \mathrm{mL}$ of diluted antiserum) (Fig. 3b); 2) omitting the neurotensin antiserum in the first incubation bath (Fig. 3e); and 3) preabsorption of anti-neurotensin with an excess $\left(10^{-7} \mathrm{M}\right)$ of synthetic substance $\mathrm{P}$, angiotensin II, beta-endorphin, somatostatin-28 (1-12), vasoactive intestinal peptide, and neuropeptide Y (Sigma-Aldrich). In all cases, the results confirmed the specificity of the antisera used in this study.

Mapping. Mapping was carried out following the frontal planes of the alpaca diencephalon that have been published in previous articles addressing the distribution of neuropeptides in alpaca's diencephalon [27-32, 34]. For each frontal plane of the alpaca diencephalon, 5-8 sections were studied. For nomenclature of the diencephalic nuclei, we used that published in the previous papers [27, 28, 32, 34]. Moreover, the brain atlas of Lama glama (available from the Mammalian Brain Collections of the University of Wisconsin, Madison, U.S.A.) was used. A series of sections contiguous to those reactive for the neuropeptide studied were routinely stained for Nissl substance with cresyl violet in order to identify and delineate the different nuclei of 
the diencephalon in which immunoreactive structures were found [27-32, 34].

As previously reported $[27,28]$, in order to determine the density of the immunoreactive fibers in alpaca's diencephalon these were graded into four categories under microscopic observation: high, moderate, low and single (a few immunoreactive fibers). This was accomplished following the protocol described by Coveñas et al. [35], and involved viewing the sections under bright-light illumination at constant magnification with reference to photographs in which the density (high, moderate and low) of the immunoreactive fibers had been established previously. Additionally, the length of the immunoreactive fibers was considered as short $(<90 \mu \mathrm{m})$, medium $(90-120 \mu \mathrm{m})$ or long $(>120 \mu \mathrm{m})$ as previously described [27]. Measurement of the size of the immunoreactive cell bodies containing neurotensin studied here was carried out as previously described [35]: cell bodies with a diameter of $<15 \mu \mathrm{m}$ were considered small; those with a diameter between 15-25 $\mu \mathrm{m}$ were medium-sized, and those with a diameter $>25 \mu \mathrm{m}$ were large. The sizes of the immunopositive cell bodies were measured using a micrometer grid with the nuclei in the focal plane. The number of immunostained cell bodies appearing in each section was counted; a high density of cell bodies was considered when we found more than 20 cell bodies/region/section; a moderate density when we found 10-20 cell bodies/region/ /section, and a low density when we found fewer than 10 cell bodies/region/section [27, 28].

Photomicrographs were obtained with an Olympus DP50 digital camera (Olympus, Tokyo, Japan) attached to a Kyowa Unilux 12 microscope (Kyowa, Tokyo, Japan). To improve the visualization of the results, only the brightness and contrast of the images were adjusted, without any further manipulation of the photographs. Adobe Photoshop CS6Software was used to view the images and adjust their brightness and contrast.

\section{Results}

\section{General considerations}

The distribution of the immunoreactive fibers and cell bodies containing neurotensin in the alpaca diencephalon is shown in Table 1 and in Figures $1-4$. Cell bodies containing neurotensin were found in the zona incerta and in the hypothalamus. In the latter structure, seven clusters of immunoreactive cell bodies were visualized. In all cases, a low/moderate density of these cell bodies was observed. In general, these cell bodies were round or fusiform and medium-large in size, showing 2-3 dendrites. The thalamus was devoid of cell bodies containing neurotensin. In both, thalamus and hypothalamus immunoreactive fibers showed a widespread distribution. In the thalamus, a high density of these fibers was mainly found in the midline nuclei, whereas in the hypothalamus a high density was in general observed by the whole structure. In general, immunoreactive fibers were thin, short or medium in length, non-branched and with varicosities. In general, in the four diencephalons used in this study, the distribution of the immunoreactive structures (fibers and cell bodies) and the density of such structures were quite similar.

\section{Immunoreactive cell bodies}

A low density of cell bodies containing neurotensin has been observed in the zona incerta (Fig. 1d, e). In the hypothalamus, a moderate density was found above the fornix (Fig. 1b, c) and in the caudal part of the lateral hypothalamic area (Fig. 1d, e), whereas a low density was observed in the anterior (Fig. 1a) and dorsal hypothalamic areas (Fig. 1c, 2b), rostral part of the lateral hypothalamic area (Fig. 1a-c, 2c, d), suprachiasmatic nucleus (Fig. 1a, 2e, f), periventricular region of the hypothalamus (Fig. 1b, c and 3b) and in the ventromedial hypothalamic nucleus (Fig. 1b, c).

\section{Immunoreactive fibers}

In the thalamus, a high density of immunoreactive fibers was observed in the central medial (Fig. 1b-e), reuniens (Fig. 1a-d, 3c), rhomboid (Fig. 1b, c, 3d), subparafascicular (Fig. 1e) and dorsal part of the reticular thalamic nuclei (Fig. 1a-d) and a moderate density in the anterodorsal thalamic nucleus (Fig. 1a, b) and in the paraventricular thalamic nucleus (Fig. 1a-e, 3e). Single immunoreactive fibers were visualized in the medial habenular nucleus (Fig. 1d, e), paracentral thalamic nucleus (Fig. 1c, d) and in the medial and lateral parts of the ventroposterior thalamic nucleus (Fig. 1c-e) and a low density in the anteromedial (Fig. 1a, b), anteroventral (Fig. 1a, b), centrolateral (Fig. 1c, d), laterodorsal (Fig. 1c-e), lateroposterior (Fig. 1d, e), ventroanterior (Fig. 1a), ventrolateral (Fig. 1b), ventromedial (Fig. 1b-d), reticular (Fig. 1a-e) and mediodorsal (Fig. 1b-e, 4d) thalamic nuclei and in the lateral habenular nucleus (Fig. 1d, e). A low density of immunoreactive fibers was also observed in the zona incerta (Fig. 1d, e, 4f).

In the hypothalamus, a high density of immunoreactive fibers containing neurotensin was found in the anterior, dorsal and lateral hypothalamic areas (Fig. 1a-e), lateral mammillary nucleus (Fig. 1e), periventricular region (Fig. 1a-e), around the fornix (Fig. 1a-d, 4b, c), posterior hypothalamic nucleus (Fig. 1d, e, 4e), median eminence (Fig. 1c), paraventricular hypothalamic nucleus (Fig. 1a, b, 3f), suprachiasmatic nucleus (Fig. 1a) and in the ventromedial hypothalamic nucleus (Fig. 1b, c). A moderate density was found in the arcuate nucleus (Fig. 1c, d) and single fibers 
Table 1. Density of fibers and cell bodies containing neurotensin in the alpaca diencephalon

\begin{tabular}{|c|c|c|c|c|c|}
\hline \multicolumn{6}{|l|}{ Hypothalamic nuclei } \\
\hline Nuclei & Cell Bodies & Fibers & Nuclei & Cell bodies & Fibers \\
\hline Anterior hypothalamic area (AHy) & + & +++ & Median eminence (ME) & - & +++ \\
\hline Arcuate nucleus (Arc) & - & ++ & Paraventricular hypothalamic nucleus (PVH) & - & +++ \\
\hline Dorsal hypothalamic area (DA) & + & +++ & Perifornical region. Above the Fornix (f) & ++ & +++ \\
\hline Fornix (f) & - & - & Periventricular area of the hypothalamus & + & +++ \\
\hline Lateral hypothalamic area (LH) & $+/++$ & +++ & Posterior hypothalamic nucleus (PH) & - & +++ \\
\hline Lateral mammillary nucleus (LM) & - & +++ & Suprachiasmatic nucleus (sch) & + & +++ \\
\hline Mammillothalamic tract (mt) & - & - & Supraoptic hypothalamic nucleus (SO) & - & s \\
\hline Medial mammillary nucleus (MM) & - & $\mathrm{s}$ & Ventromedial hypothalamic nucleus (VMH) & + & +++ \\
\hline \multicolumn{6}{|l|}{ Thalamic nuclei } \\
\hline Nuclei & Cell bodies & Fibers & Nuclei & Cell bodies & Fibers \\
\hline Anterodorsal thalamic nucleus (AD) & - & ++ & Reticular thalamic nucleus (Rt) & - & $+/+++$ \\
\hline Anteromedial thalamic nucleus (AM) & - & + & Reuniens thalamic nucleus $(\mathrm{Re})$ & - & +++ \\
\hline Anteroventral thalamic nucleus (AV) & - & + & Rhomboid thalamic nucleus (Rh) & - & +++ \\
\hline Central medial thalamic nucleus (CM) & - & +++ & Stria medullaris (sm) & - & - \\
\hline Centrolateral thalamic nucleus (CL) & - & + & Subparafascicular thalamic nucleus (SPF) & - & +++ \\
\hline Lateral geniculate nucleus (LG) & - & - & Subthalamic nucleus (STh) & - & $\mathrm{s}$ \\
\hline Lateral habenular nucleus (LHb) & - & + & Ventroanterior thalamic nucleus (VA) & - & + \\
\hline Laterodorsal thalamic nucleus (LD) & - & + & Ventrolateral thalamic nucleus (VL) & - & + \\
\hline Lateroposterior thalamic nucleus (LP) & - & + & Ventromedial thalamic nucleus (VM) & - & + \\
\hline Medial habenular nucleus (MHb) & - & $\mathrm{s}$ & Ventroposterior thalamic nucleus, lateral part (VPL) & - & $\mathrm{s}$ \\
\hline Mediodorsal thalamic nucleus (MD) & - & + & Ventroposterior thalamic nucleus, medial part (VPM) & - & $\mathrm{s}$ \\
\hline Paracentral thalamic nucleus (PC) & - & $\mathrm{s}$ & Zona incerta (ZI) & + & + \\
\hline Paraventricular thalamic nucleus (PVA) & - & ++ & & & \\
\hline
\end{tabular}

Cell bodies ( +- low density; ++- moderate density); Fibers ( - single fibers; +- low density; ++- moderate density; +++- high density). - denotes lack of immunoreactivity.

in the medial mammillary nucleus (Fig. 1e) and the supraoptic hypothalamic nucleus (Fig. 1a, b).

\section{Discussion}

This is the first report describing the distribution of immunoreactive structures containing neurotensin in the alpaca diencephalon. The neuroanatomical findings reported here add to our current knowledge about the neurotensinergic system in the camelid central nervous system, since the mapping of neurotensin-immunoreactive fibers and cell bodies in the alpaca brainstem has been carried out previously [12]. The origin of the immunoreactive fibers in the alpaca diencephalon is unknown as well as we have no data indicating whether the neurotensin-immunoreactive cell bodies observed in the alpaca diencephalon are local and/or projecting neurons. However, our data suggest that many neurons could be projecting neurons, since a widespread distribution of immunoreactive fibers containing neurotensin has been observed. It is important to note that this study was carried out in animals not treated with colchicine and it is known that sometimes the visualization of a widespread distribution of peptidergic cell bodies only occurred in colchicine-treated animals [30]. Because the administration of colchicine in alpacas must be ruled out for ethical reasons, other techniques (e.g., in situ hybridization) must be used to elucidate the complete distribution of neurons containing neurotensin in the alpaca diencephalon.

The widespread distribution of the neurotensinergic system observed in the alpaca diencephalon suggests that the neuropeptide might be involved in many physiological mechanisms, as it has been reported in other mammals. Thus, neurotensin would be involved 


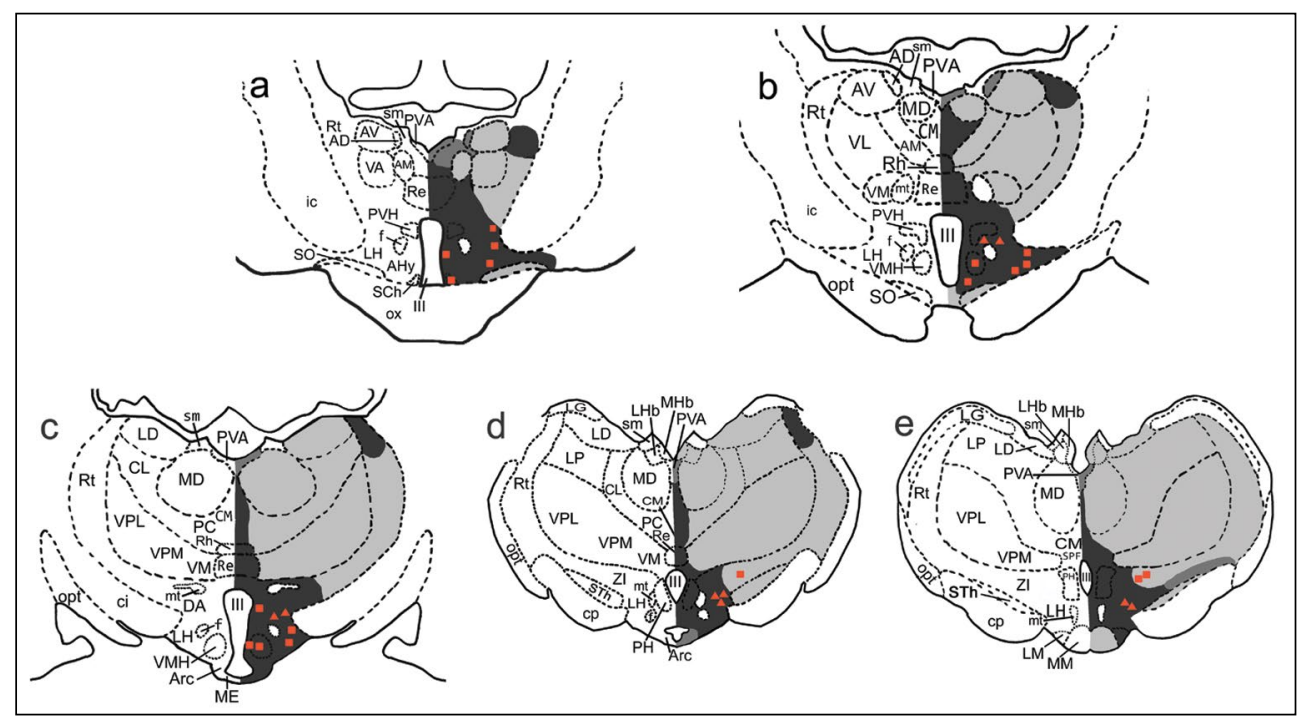

Figure 1. Distribution of neurotensin-immunoreactive fibers and cell bodies in frontal planes of the alpaca diencephalon from rostral (a) to caudal levels (e). Cell bodies are represented by closed squares (low density) and triangles (moderate density). Immunoreactive fibers are represented by slightly dark (low density or single fibers), moderately dark (moderate density) and strongly dark (high density). Abbreviations: III — third ventricle; AD — anterodorsal thalamic nucleus; AHy — anterior hypothalamic area; AM — anteromedial thalamic nucleus; Arc — arcuate nucleus; AV — anteroventral thalamic nucleus; $\mathrm{CL}$ - centrolateral thalamic nucleus; $\mathrm{CM}$ - central medial thalamic nucleus; cp — cerebral peduncle; DA — dorsal hypothalamic area; f — fornix; ic — capsula interna; LD — laterodorsal thalamic nucleus; LG — lateral geniculate nucleus; LH — lateral hypothalamic area; LHb - lateral habenular nucleus; LM — lateral mammillary nucleus; LP — lateroposterior thalamic nucleus; $\mathrm{MD}$ - mediodorsal thalamic nucleus; $\mathrm{ME}$ - median eminence; $\mathrm{MHb}$ - medial habenular nucleus; MM — medial mammillary nucleus; $\mathrm{mt}$ — mammillothalamic tract; opt — optic tract; ox — optic chiasm; PC — paracentral thalamic nucleus; $\mathrm{PH}$ - posterior hypothalamic nucleus; PVA — paraventricular thalamic nucleus; $\mathrm{PVH}$ - paraventricular hypothalamic nucleus; $\mathrm{Re}$ - reuniens thalamic nucleus; Rh — rhomboid thalamic nucleus; Rt — reticular thalamic nucleus; sch - suprachiasmatic nucleus; sm — stria medullaris; SO — supraoptic hypothalamic nucleus; SPF — subparafascicular thalamic nucleus; STh — subthalamic nucleus; VA — ventroanterior thalamic nucleus; VL — ventrolateral thalamic nucleus; VM - ventromedial thalamic nucleus; VMH - ventromedial hypothalamic nucleus; VPL — ventroposterior thalamic nucleus, lateral part; VPM - ventroposterior thalamic nucleus, medial part; ZI — zona incerta.

in feeding, sexual activity, vigilance behavior, homeostasis, thermogenesis and neuroendocrine mechanisms $[3,5,8,13]$. Future studies should be focused on such possible functions and on the distribution of neurotensin receptors in the alpaca diencephalon.

\section{Neuropeptides in the alpaca diencephalon}

In order to compare adequately the distribution of neuropeptides in the alpaca diencephalon, here male alpacas were only used, since in previous works in which the distribution of neuropeptides was carried out; male animals were exclusively used [27-32]. Moreover, all animals, used here and in the previous studies, were always maintained at $0 \mathrm{~m}$ on the sea level (from birth to death) and were not treated with colchicine. The distribution of immunoreactive structures containing calcitonin gene-related peptide (CGRP), adrenocorticotropic hormone (ACTH), leucine-enkephalin, beta-endorphin (1-27), alpha-melanocyte-stimulating hormone $(\alpha \mathrm{MSH})$ or alpha-neo-endorphin and somatostatin-28 (1-12) has been previously reported in the alpaca diencephalon $[27,28,32]$.

In all the thalamic and hypothalamic nuclei in which we have observed neurotensin-immunoreactive fibers, the presence of fibers containing beta-endorphin (1-27), $\alpha \mathrm{MSH}$, alpha-neo-endorphin or somatostatin-28 (1-12) has been also reported [27, 28, 32]. Moreover, fibers containing CGRP, ACTH, or leucine-enkephalin $[28,32]$ were observed in all the hypothalamic nuclei in which we have visualized neurotensin-immunoreactive fibers. Thus, there is a close anatomical relationship in the alpaca diencephalon between the above-mentioned neuropeptides. Moreover, the data suggest a possible functional interaction between these neuropeptides and a possible coexistence of them.

Our study shows for the first time in the alpaca diencephalon the presence of peptidergic cell bodies in the suprachiasmatic nucleus and zona incerta. 

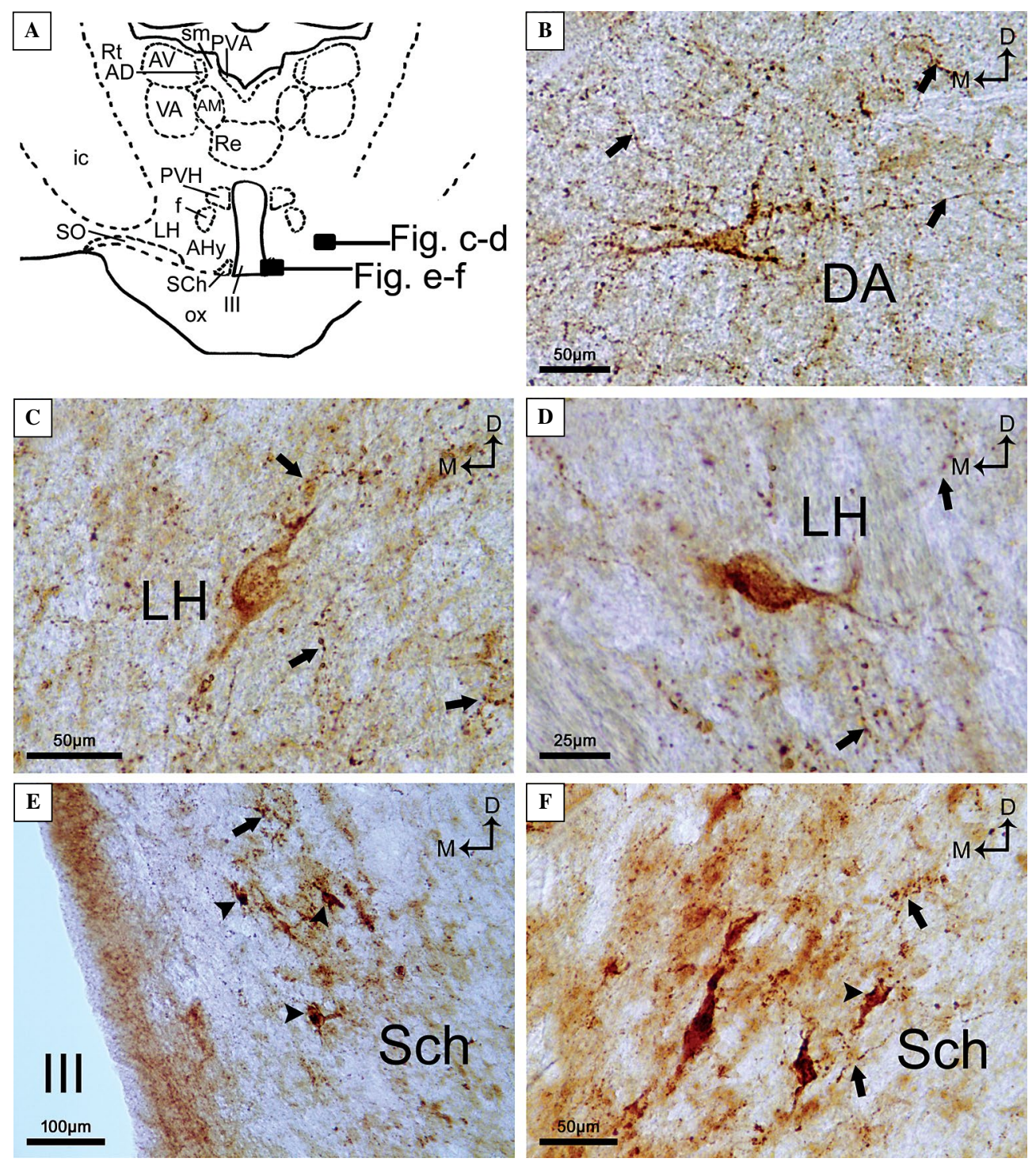

Figure 2. Neurotensin-immunoreactive cell bodies (arrowheads) in the alpaca hypothalamus. (A) Frontal section of the diencephalon at the level of the optic chiasm (ox). For nomenclature of the nuclei and tracts, see list of abbreviations in Figure 1. The photographs shown in $\mathbf{C}-\mathbf{F}$ were respectively taken from the regions delimited by the rectangles in $\mathbf{A}$ (indicated as Fig. $\mathrm{c}-\mathrm{d}$ and Fig. e-f). Cell bodies containing the peptide located in (B) the dorsal hypothalamic area (DA) (see Fig. 1c), (C, D) the lateral hypothalamic area (LH) and (E, F) in the suprachiasmatic nucleus (Sch). Arrows: immunoreactive fibers.

In the lateral hypothalamic area and in the ventromedial hypothalamic nucleus, cell bodies containing neurotensin, $\alpha \mathrm{MSH}$, alpha-neo-endorphin, somatostatin-28 (1-12) or CGRP have been reported [27, 28, 32]. According to the morphological characteristics (e.g., size, shape) of the peptidergic neurons located in both hypothalamic nuclei $[27,28,32]$, it seems that a possible coexistence of these neuropeptides could occur. The administration of CGRP into the ventromedial hypothalamic nucleus increases the temperature of the interscapular brown adipose tissue, heart rate, oxygen consumption and colonic temperature [36]. In the anterior and dorsal hypothalamic areas, cell bodies containing neurotensin or CGRP have been also located [28]. In future studies, the coexistence of both neuropeptides should be addressed in the anterior and dorsal hypothalamic areas.

\section{Neurotensin in the mammalian diencephalon}

The distribution of fibers and cell bodies containing neurotensin has been carried out studied in rat, cat and human diencephalons [3, 5, 8, 11]. It should be noted that, here, we have used the same anti-neurotensin antiserum and applied the same immunohistochemical method that in the study carried out in the feline [3]. In general, in the hypothalamus, the neurotensinergic 

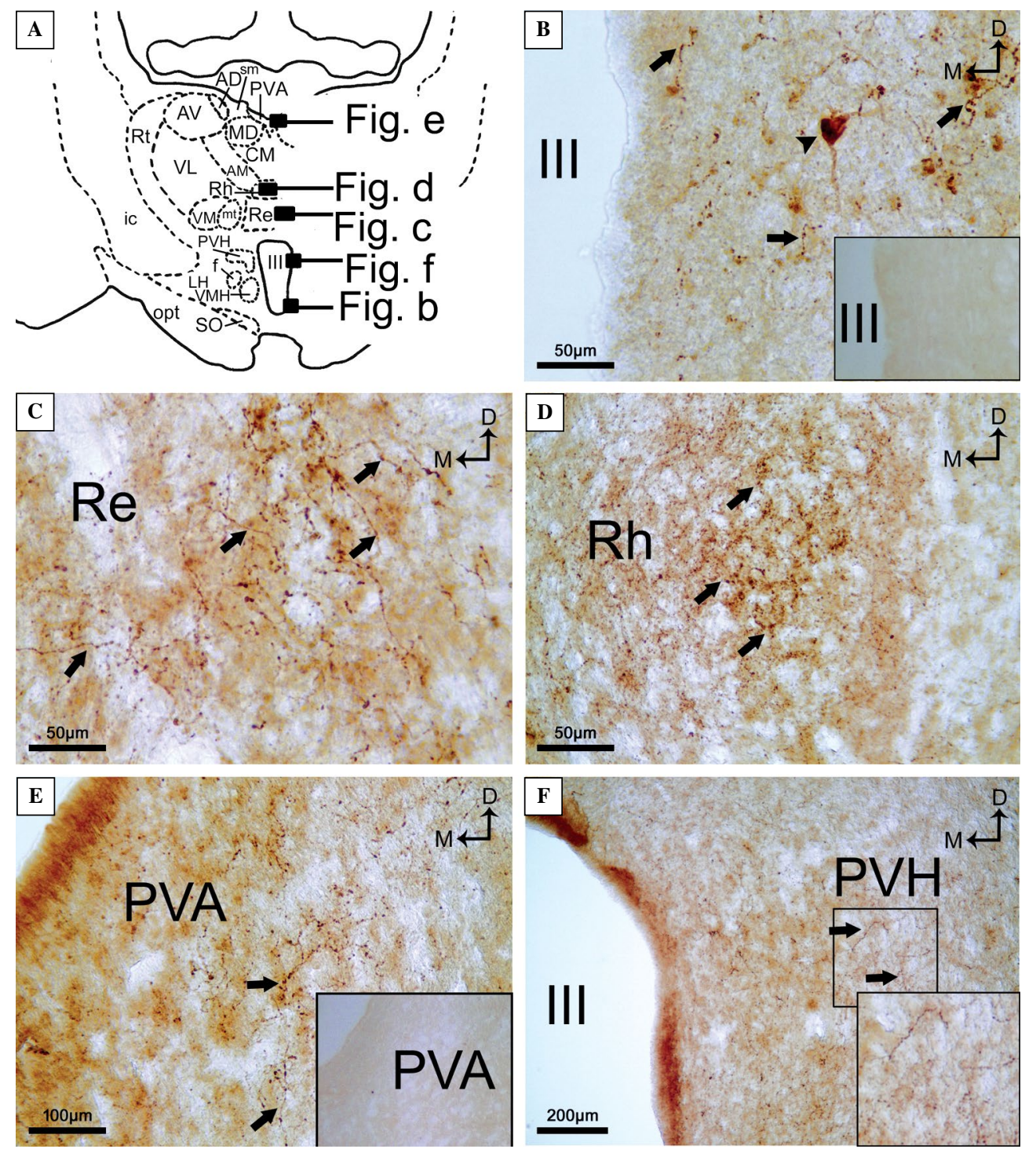

Figure 3. Neurotensin-immunoreactive cell bodies (arrowhead) and fibers (arrows) in the alpaca diencephalon. (A) Frontal section of the diencephalon at the level of the optic tract (opt). For nomenclature of the nuclei and tracts, see list of abbreviations in Figure 1. The photographs shown in B-F were respectively taken from the regions delimited by the rectangles in a (indicated as Fig. B-F). (B) Immunoreactive cell body located in the periventricular region of the hypothalamus. Inset: absence of immunoreactivity in the periventricular region of the hypothalamus when the preabsorption of the anti-neurotensin antibody was carried out with neurotensin. Immunoreactive fibers in the reuniens thalamic nucleus $(\mathrm{Re})(\mathbf{C})$, rhomboid thalamic nucleus (D), paraventricular thalamic nucleus (PVA) (E) (inset in (e): absence of immunoreactivity in the paraventricular thalamic nucleus (PVA) when the anti-neurotensin antibody was omitted) and in the paraventricular hypothalamic nucleus (F) (inset at bottom right: a higher magnification of the upper rectangle).

innervation in rat, cat, alpaca and humans is quite similar since in these four species neurotensin-immunoreactive fibers have been found through the whole hypothalamus $[3,5,8,11]$. Moreover, in the four species neurotensin-immunoreactive fibers have been observed in the thalamus along the midline $[3,5,8,11]$. However, in rat, cat and humans, neurotensin-immunoreactive fibers were totally absent in the lateral region of the thalamus (e.g., reticular thalamic nucleus) [3, 5 ,
8,11 , but in the alpaca we observed immunoreactive fibers containing neurotensin in the lateral thalamic nuclei. This means that, among the mammalian thalamus, the alpaca shows the most widespread distribution of neurotensin-immunoreactive fibers. It seems that this discrepancy is due to species differences and suggests that neurotensin could be involved in special and unique regulatory mechanisms in the lateral thalamic nuclei of the alpaca. 

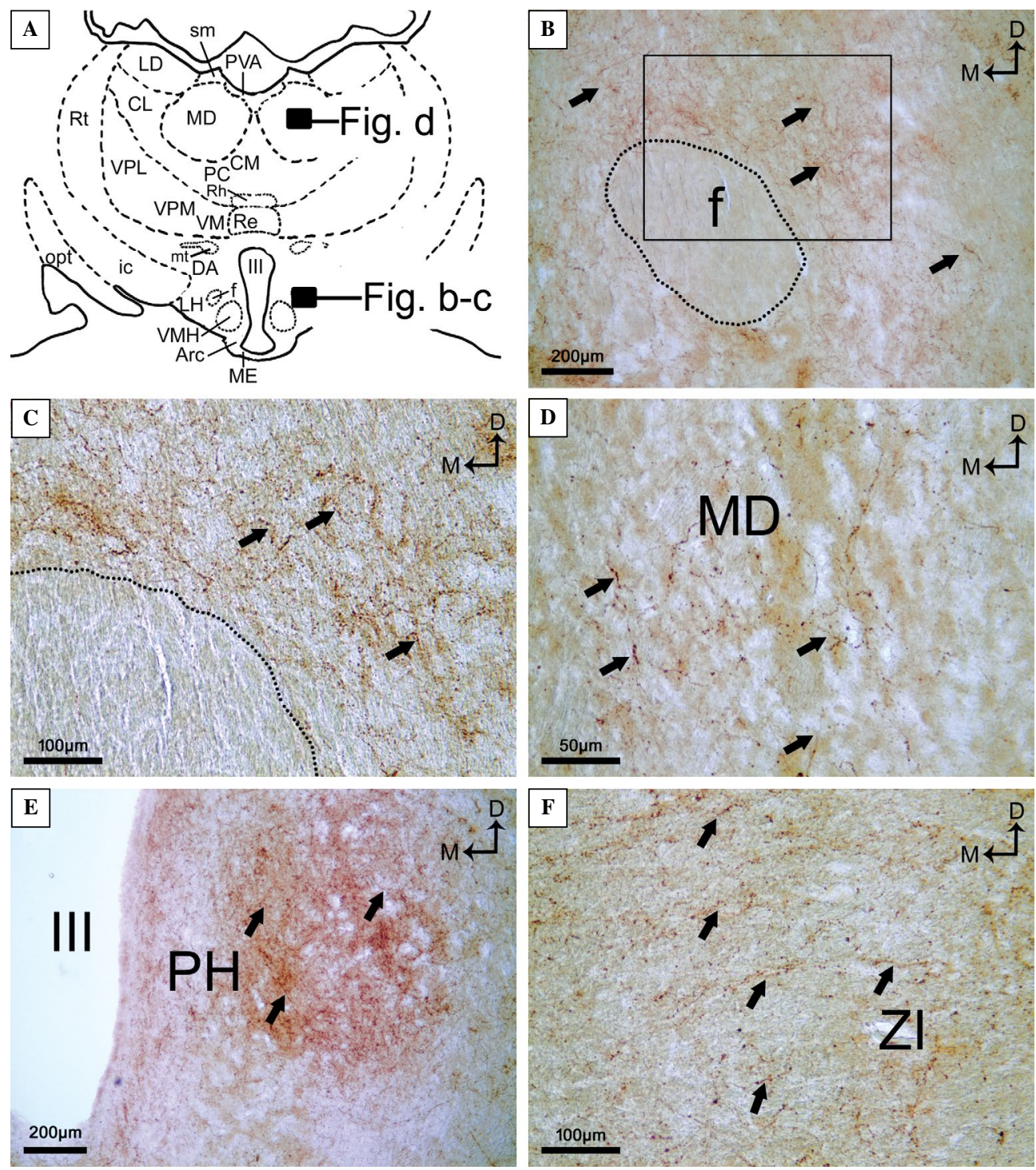

Figure 4. Neurotensin-immunoreactive fibers (arrows) in the alpaca diencephalon. (A) Frontal section of the diencephalon at the level of the median eminence (ME). For nomenclature of the nuclei and tracts, see list of abbreviations in Figure 1. The photographs shown in B-D were respectively taken from the regions delimited by the rectangles in a (indicated as Fig. b-c and Fig. d). (B) Immunoreactive fibers around the fornix (f). (C) A higher magnification of the region delimited by the rectangle in B. Fibers containing the peptide located in the (D) mediodorsal thalamic nucleus (MD), (E) posterior hypothalamic nucleus (PH) (see Fig. 1d, e) and (F) in the zona incerta (ZI) (see Fig. 1d, e).

Regarding the distribution of thalamic neurotensin-immunoreactive cell bodies, they are more widely distributed in cat than in rat, alpaca and human [3, $5,8,11]$. In the two latter species, neurotensin-immunoreactive cell bodies were not observed [8]. This discrepancy could be due to technical considerations, since in both rats and cats intraventricular injections of colchicine were carried out $[5,11]$. Regarding the hypothalamus, rats, cats and humans $[3,5,8,11]$ showed a more widespread distribution of neurotensin-immunoreactive cell bodies than that observed in the alpaca. In these four species, cell bodies containing neurotensin were found in the lateral hypothalamic area, ventromedial hypothalamic nucleus and in the suprachiasmatic nucleus. In the arcuate nucleus, neurotensin-immunoreactive cell bodies have been visualized in rat, cat and humans, but not in alpacas. The discrepancy in the distribution of cell bodies containing neurotensin in the mammalian hypothalamus could be due to technical considerations (e.g., primary antisera used, administration of colchicine) and/or species differences.

In summary, this study increases our knowledge of the chemical neuroanatomy of neurotensin in 
camelids, since a previous study described the distribution of immunoreactive structures (cell bodies and fibers) containing neurotensin in the alpaca brainstem [12]. The mapping of neurotensin in the alpaca diencephalon may help to better understand the involvement of this neuropeptide in its multiple physiological actions.

\section{Acknowledgements}

The authors wish to thanks Professor Gérard Tramu (Université de Bordeaux I, France) for the gift of the primary antibody and Mr. Nicholas Skinner (University of Salamanca, Spain) for supervising the English text. This study was supported by Ministerio de Economía y Competitividad (SAF2016-79008-P), Spain and Programa Nacional de Innovación para la Competitividad y Productividad, Innóvate Perú (CONV-000116-2015-FONDECYT-DE), Perú.

\section{References}

1. Carraway RE, Leeman SE. Characterization of radioimmunoassayable neurotensin in the rat. Its differential distribution in the central nervous system, small intestine and stomach. J Biol Chem 1976; 251: 7045-7052, indexed in Pubmed: 993203.

2. Cooper PE, Fernstrom MH, Rorstad OP, et al. The regional distribution of somatostatin, substance $P$ and neurotensin in human brain. Brain Res. 1981; 218(1-2): 219-232, indexed in Pubmed: 6168327.

3. De León M, Coveñas R, Narváez JA, et al. Neurotensin-like immunoreactivity in the diencephalon of the adult male cat. Peptides. 1991; 12(2): 257-264, indexed in Pubmed: 2067978.

4. De León M, Coveñas R, Narváez JA, et al. Distribution of neurotensin-like immunoreactive cell bodies and fibers in the brainstem of the adult male cat. Peptides. 1991; 12(6): 1201-1209, indexed in Pubmed: 1815208.

5. Jennes L, Stumpf WE, Kalivas PW. Neurotensin: topographical distribution in rat brain by immunohistochemistry. J Comp Neurol. 1982; 210(3): 211-224, doi: 10.1002/cne.902100302, indexed in Pubmed: 6754769.

6. Kataoka K, Mizuno N, Frohman LA. Regional distribution of immunoreactive neurotension in monkey brain. Brain Res Bull. 1979; 4(1): 57-60, indexed in Pubmed: 111778.

7. Kobayashi RM, Brown M, Vale W. Regional distribution of neurotensin and somatostatin in rat brain. Brain Res. 1977; 126(3): 584-588, indexed in Pubmed: 861744.

8. Mai JK, Triepel J, Metz J. Neurotensin in the human brain. Neuroscience. 1987; 22(2): 499-524, indexed in Pubmed: 3670596.

9. Sánchez ML, Vecino E, Coveñas R. Distribution of neurotensin and somatostatin-28 (1-12) in the Minipig Brainstem. Anat Histol Embryol. 2016; 45(4): 260-276, doi: 10.1111/ahe.12194, indexed in Pubmed: 26250798.

10. Triepel J, Weindl A, Heinrich D, et al. Distribution of neurotensin-immunoreactive perikarya in the brainstem of the guinea pig. Histochemistry. 1984; 13: 229-312, doi: 10.1111/ /ahe.12194.

11. Uhl GR, Kuhar MJ, Snyder SH. Neurotensin: immunohistochemical localization in rat central nervous system. Proc Natl Acad Sci USA. 1977; 74(9): 4059-4063, doi: 10.1073/ /pnas.74.9.4059.
12. de Souza E, Aguilar LA, Díaz-Cabiale Z, et al. Mapping of neurotensin in the alpaca (Lama pacos) brainstem. Anat Histol Embryol. 2014; 43(4): 245-256, doi: 10.1111/ahe.12067, indexed in Pubmed: 23692174.

13. Schroeder LE, Leinninger GM. Role of central neurotensin in regulating feeding: Implications for the development and treatment of body weight disorders. Biochim Biophys Acta. 2018; 1864(3): 900-916, doi: 10.1016/j.bbadis.2017.12.036, indexed in Pubmed: 29288794.

14. Kaczyńska K, Zając D, Wojciechowski P, et al. Neuropeptides and breathing in health and disease. Pulm Pharmacol Ther. 2018; 48: 217-224, doi: 10.1016/j.pupt.2017.12.001, indexed in Pubmed: 29223509.

15. Kuhre RE, Christiansen CB, Saltiel MY, et al. On the relationship between glucose absorption and glucose-stimulated secretion of GLP-1, neurotensin, and PYY from different intestinal segments in the rat. Physiol Rep. 2017; 5(23), doi: 10.14814/phy2.13507, indexed in Pubmed: 29199179.

16. Lénárd L, László $\mathrm{K}$, Kertes E, et al. Substance $\mathrm{P}$ and neurotensin in the limbic system: Their roles in reinforcement and memory consolidation. Neurosci Biobehav Rev. 2018; 85: 1-20, doi: 10.1016/j.neubiorev.2017.09.003, indexed in Pubmed: 28887225.

17. Gandou C, Ohtani A, Senzaki K, et al. Neurotensin promotes the dendrite elongation and the dendritic spine maturation of the cerebral cortex in vitro. Neurosci Res. 2010; 66(3): 246-255, doi: 10.1016/j.neures.2009.11.007, indexed in Pubmed: 19941912.

18. Martorana A, Martella G, D'Angelo V, et al. Neurotensin effects on $\mathrm{N}$-type calcium currents among rat pallidal neurons: an electrophysiological and immunohistochemical study. Synapse. 2006; 60(5): 371-383, doi: 10.1002/syn.20306, indexed in Pubmed: 16838364.

19. Kim J, Napier D, Weiss H, et al. Neurotensin receptor 3/Sortilin Contributes to Tumorigenesis of Neuroendocrine Tumors Through Augmentation of Cell Adhesion and Migration. Neoplasia. 2018; 20(2): 175-181, doi: 10.1016/j.neo.2017.11.012.

20. Abbaci A, Talbot H, Saada S, et al. $<$ sup $>177<$ /supl $>$ for neurotensin receptor type 2 protects B-cell chronic lymphocytic leukemia cells from apoptosis. Oncogene. 2017; 37(6): 756-767, doi: 10.1038/onc.2017.365.

21. Baum RP, Singh A, Schuchardt C, et al. Lu-3BP-227 for neurotensin receptor 1-targeted therapy of metastatic pancreatic adenocarcinoma - first clinical results. J Nucl Med. 2017 [Epub ahead of print], doi: 10.2967/jnumed.117.193847, indexed in Pubmed: 29025990.

22. Bravo PW, Stewart DR, Lasley BL, et al. Hormonal indicators of pregnancy in llamas and alpacas. J Am Vet Med Assoc. 1996; 208(12): 2027-2030, indexed in Pubmed: 8707678.

23. Correa JE, Ratto MH, Gatica R. Superovulation in llamas (Lama glama) with pFSH and equine chorionic gonadotrophin used individually or in combination. Anim Reprod Sci. 1997; 46(3-4): 289-296, indexed in Pubmed: 9231267.

24. Ratto MH, Gatica R, Correa JE. Timing of mating and ovarian response in llamas (Lama glama) treated with pFSH. Anim Reprod Sci. 1997; 48(2-4): 325-330, indexed in Pubmed: 9452884

25. Ratto MH, Huanca W, Singh J, et al. Local versus systemic effect of ovulation-inducing factor in the seminal plasma of alpacas. Reprod Biol Endocrinol. 2005; 3: 29, doi: 10.1186/1477-7827-3-29, indexed in Pubmed: 16018817.

26. Ratto M, Huanca W, Singh J, et al. Comparison of the effect of natural mating, $\mathrm{LH}$, and $\mathrm{GnRH}$ on interval to ovulation and luteal function in llamas. Anim Reprod Sci. 2006; 91(3-4): 
299-306, doi: 10.1016/j.anireprosci.2005.03.015, indexed in Pubmed: 15896931.

27. Coveñas R, Mangas A, Medina LE, et al. Mapping of somatostatin-28 (1-12) in the alpaca diencephalon. J Chem Neuroanat. 2011; 42(1): 89-98, doi: 10.1016/j.jchemneu.2011.06.006, indexed in Pubmed: 21729751.

28. Coveñas R, Sánchez ML, Mangas A, et al. Mapping of CGRP in the alpaca diencephalon. J Chem Neuroanat. 2012; 45(1-2): 36-44, doi: 10.1016/j.jchemneu.2012.07.004, indexed in $\mathrm{Pu}-$ bmed: 22922318.

29. de Souza E, Yi P, Aguilar LA, et al. Mapping of leucine-enkephalin in the alpaca (Lama pacos) brainstem. In: Coveñas R, Mangas A, Narváez JA, eds. Focus on Neuropeptide Research. Trivandrum: Transworld Research Network. 2007: 103-114.

30. de Souza E, Coveñas R, Yi P, et al. Mapping of CGRP in the alpaca (Lama pacos) brainstem. J Chem Neuroanat. 2008; 35(4): 346-355, doi: 10.1016/j.jchemneu.2008.02.004, indexed in Pubmed: 18420379.

31. Souza EDe, Sánchez M, Aguilar L, et al. Mapping of somatostatin-28 (1-12) in the alpaca (Lama pacos) brainstem. Microsc Res and Tech. 2015; 78(5): 363-374, doi: 10.1002/jemt.22482.
32. Manso B, Sánchez ML, Medina LE, et al. Immunohistochemical mapping of pro-opiomelanocortin- and pro-dynorphin-derived peptides in the alpaca (Lama pacos) diencephalon. J Chem Neuroanat. 2014; 59-60: 36-50, doi: 10.1016/j. jchemneu.2014.06.001, indexed in Pubmed: 24956196.

33. Studler JM, Kitabgi P, Tramu G, et al. Extensive co-localization of neurotensin with dopamine in rat meso-cortico-frontal dopaminergic neurons. Neuropeptides. 1988; 11(3): 95-100, indexed in Pubmed: 3133572.

34. Marcos P, Arroyo-Jiménez MM, Lozano G, et al. Mapping of tyrosine hydroxylase in the diencephalon of alpaca (Lama pacos) and co-distribution with somatostatin-28 (1-12). J Chem Neuroanat. 2013; 50-51: 66-74, doi: 10.1016/j. jchemneu.2013.02.006, indexed in Pubmed: 23474224.

35. Coveñas R, de Le, Narváez JA, et al. Anatomical distribution of beta-endorphin (1-27) in the cat brainstem: an immunocytochemical study. Anat Embryol. 1999; 199: 161-167. PMID. : 9930622

36. Kobayashi A, Osaka T, Namba Y, et al. CGRP microinjection into the ventromedial or dorsomedial hypothalamic nucleus activates heat production. Brain Res. 1999; 827(1-2): 176-184, indexed in Pubmed: 10320707.

Submitted: 20 April, 2017 Accepted after reviews: 26 February, 2018 Available as AoP: 5 March, 2018 